With compliments of the Author 


\title{
Mild Two-Step Conversion of Primary Amides to Alcohols by Reduction of Acylimidodicarbonates with Sodium Borohydride
}

\author{
Ulf Ragnarsson, ${ }^{* a}$ Leif Grehn, ${ }^{a}$ Luis S. Monteiro, ${ }^{b}$ Hernani L. S. Maia ${ }^{b}$ \\ a Department of Biochemistry, University of Uppsala, Biomedical Center, PO Box 576, SE-751 23 Uppsala, Sweden \\ Fax +46(18)552139; E-mail: urbki@bmc.uu.se \\ b Departamento de Quimica, Universidade do Minho, Gualtar, P-4700-320 Braga, Portugal \\ Received 8 September 2003
}

\begin{abstract}
Di-tert-butyl acylimidodicarbonates, prepared from primary amides with two equivalents of di-tert-butyl dicarbonate under catalysis by 4-dimethylaminopyridine, readily undergo selective reductive cleavage of their acyl CO-N bonds by $\mathrm{NaBH}_{4}$ to provide the corresponding alcohols in high yields.
\end{abstract}

Key words: alcohols, amides, cleavage, reductions, sodium borohydride

Recently, in connection with studies of model $N$-benzyl acylcarbamates we observed that these substances underwent regiospecific acyl $\mathrm{CO}-\mathrm{N}$ reductive cleavage under relatively mild conditions. ${ }^{1}$ In that context it occurred to us that this reaction might be exploited for synthetic purposes. As a continuation, we now present a simple two-step procedure for conversion of primary amides to alcohols based on similar principles. Rather few transformations of this kind involving hydrogenation, electrochemical and dissolving metal reduction and metal hydride reagents have been reported in the literature. ${ }^{2}$ Some tertiary amides have recently been shown to undergo notably mild reductive cleavage to alcohols by metal hydride reagents. ${ }^{3}$

As first shown by Grieco et al., secondary amides and lactams undergo facile reaction with di-tert-butyl dicarbonate $\left(\mathrm{Boc}_{2} \mathrm{O}\right)$ in the presence of 4-dimethylaminopyridine ${ }^{4}$ (DMAP) with formation of the corresponding acylcarbamates. ${ }^{5}$ Such compounds react with nucleophiles under cleavage of their acyl-N bonds, as a consequence of which the original amides can be converted to carbamates in two steps. Similarly, primary amides $\mathbf{1}$ undergo substitution with two equivalents of $\mathrm{Boc}_{2} \mathrm{O}$ in the presence of this catalyst to give di-tert-butyl acylimidodicarbonates $2{ }^{6}$

Compounds 2 behave as acylating agents, ${ }^{7}$ indicating a significant further electron-withdrawing effect by the second Boc group on nitrogen in comparison with that in

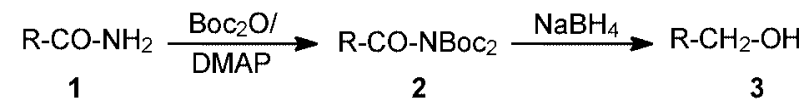

Scheme 1 Two-step conversion of primary amides via di-tert-butyl acylimidodicarbonates to alcohols

SYNLETT 2003, No. 15, pp 2386-2388

Advanced online publication: 07.11.2003

DOI: 10.1055/s-2003-42116; Art ID: G22203ST

(c) Georg Thieme Verlag Stuttgart · New York acylcarbamates. This effect has been measured for $\mathbf{2 a}$ $(\mathrm{R}=\mathrm{Ph})$ using cyclic voltammetry $(\mathrm{CV}) .^{1 \mathrm{~b}}$

To study the reactivity of di-tert-butyl acylimidodicarbonates $\mathbf{2}$ in more detail with respect to reduction, several compounds $2 \mathbf{a}-\mathbf{f}$ listed in Table 1 have been prepared from the corresponding amides $1 .^{6}$ According to TLC the syntheses of all went to completion within a maximum of 24 hours, which eliminated the need for chromatographic purification of the products. These were all obtained as highly lipophilic solids, some of which like 2e dissolved easily in light petroleum and therefore occasionally were difficult to recover by recrystallization. The variable yields for individual compounds 2 reflect this fact rather than low reactivity of some amides.

As a prelude to the preparative reduction experiments, attempts were made to characterize the acylimidodicarbonates $\mathbf{2}$ by $\mathrm{CV}$. These experiments were performed in DMF as described elsewhere. ${ }^{8}$ Although the activation potential could not be determined for all compounds, in two cases (for $\mathbf{2 a}$ and $\mathbf{2 d}$ ) we could demonstrate that the introduction of a second Boc group shifted the peaks by over $0.35 \mathrm{~V}$ to less negative potential in comparison with the corresponding $N$-benzyl acylcarbamates (Table 1). ${ }^{\text {1a,8 }}$

Compounds $\mathbf{2}$ were reduced to alcohols $\mathbf{3}$ using an excess of $\mathrm{NaBH}_{4}$ in EtOH on a 2-3 mmol scale with the simultaneous formation of an equivalent of $\mathrm{HNBOc}_{2} .{ }^{9}$ Although, as demonstrated, the latter compound could generally be efficiently separated from the desired alcohols by chromatography on silica gel, in other cases due to the high lipophilicity of the products this turned out to be more difficult and required rechromatography to provide pure products, all of which have been described before. All reductions with $\mathrm{NaBH}_{4}$ could be carried out at room temperature except in the case of 2c. As reflected by its much lower activation potential in comparison with $\mathbf{2 a}$ and $\mathbf{2 d}$ (Table 1), it was less reactive and moderate heating was therefore required. In this experiment also a side-product, formed by the alternative cleavage of a Boc-group from 2c, could be isolated. Another triacylated ammonia derivative, $\mathrm{NBoc}_{3}$, known to undergo aminolysis, ${ }^{10}$ is also cleaved to $\mathrm{HNBoc}_{2}$ by $\mathrm{NaBH}_{4}$ with high selectivity, which exemplifies the dramatic shift in stability of Boc in these compounds in comparison with simple carbamates. ${ }^{11}$ Attempts to reduce $2 \mathrm{c}$ by cathodic electrolysis ${ }^{8}$ furnished a complex mixture containing $\mathrm{HNBoc}_{2}$ but only small amounts of $\mathbf{3 c}$. 
Reduction of the proline amide derivative $2 \mathbf{f}$ with both diisobutylaluminium hydride and tri-tert-butoxy-aluminium hydride has previously been attempted but only resulted in a meager $(<10 \%)$ yield of a mixture of the corresponding aldehyde and alcohol. ${ }^{7}$

Concerning the scope of this two-step transformation (Scheme 1), it can be mentioned that several amides $\mathbf{1}$ have been converted to di-tert-butyl acylimidodicarbonates ${ }^{7,12} 2$ using Boc $_{2} \mathrm{O} / \mathrm{DMAP}$, so a fair amount of information is already available with respect to the first step. Among these amides are such containing additional ester, carbamate, alcohol and amino functions, some of which undergo additional reaction. As most functional groups other than aldehydes and ketones are stable to $\mathrm{NaBH}_{4},{ }^{13}$ the corresponding acylimidodicarbonates should therefore normally be compatible with the mild reducing agent used in the second step and provide alcohols accordingly.

Table 1 Synthesis of di-tert-Butyl Acylimidodicarbonates 2 and their Reduction to Alcohols $\mathbf{3}$ by $\mathrm{NaBH}_{4}$

\begin{tabular}{|c|c|c|c|c|}
\hline $\begin{array}{l}\text { Amide 1/ } \\
\text { Solvent }^{\mathrm{c}}\end{array}$ & $\begin{array}{l}2 \\
\text { Yield }^{\text {a }}(\%) \\
\mathrm{Mp}\left({ }^{\circ} \mathrm{C}\right) \\
-E_{\mathrm{P}} / \mathrm{V}\end{array}$ & Spectral data of $2^{\mathrm{b}}$ & $\begin{array}{l}3 \\
\text { Yield }^{\mathrm{a}} \\
(\%) \\
\mathrm{Mp}\left({ }^{\circ} \mathrm{C}\right)\end{array}$ & Spectral data of $\mathbf{3}^{\mathrm{b}}$ \\
\hline Benzamide (a)/A & $\begin{array}{l}77^{\mathrm{d}} \\
59.5-60.5 \\
1.56^{\mathrm{f}}\end{array}$ & $\begin{array}{l}\delta=1.38(18 \mathrm{H}), 7.47(\mathrm{p} \mathrm{t}, J=7 \mathrm{~Hz}, 2 \mathrm{H}) \\
7.60(\mathrm{p} \mathrm{t}, J=7 \mathrm{~Hz}, 1 \mathrm{H}), 7.83(\mathrm{p} \mathrm{d}, J=7 \\
\mathrm{Hz}, 2 \mathrm{H}) ; 1765,1740,1702 \mathrm{~cm}^{-1}\end{array}$ & $\begin{array}{l}\text { n.d.e } \\
\text { oil }\end{array}$ & $\begin{array}{l}\delta=1.96(\mathrm{br}, 1 \mathrm{H}), 4.66(2 \mathrm{H}), 7.26-7.39(\mathrm{~m}, \\
5 \mathrm{H}) ; \text { ca. } 3338(\mathrm{br}), 1454,1021,735,697 \mathrm{~cm}^{-1}\end{array}$ \\
\hline $\begin{array}{l}\text { 4-Nitrobenzamide } \\
\text { (b)/A }\end{array}$ & $\begin{array}{l}79^{g} \\
74.5-75.5 \\
0.60,1.01\end{array}$ & $\begin{array}{l}\delta=1.43(18 \mathrm{H}), 7.94(\mathrm{p} \mathrm{d}, J=8.4 \mathrm{~Hz}, 2 \\
\mathrm{H}), 8.32(\mathrm{p} \mathrm{d}, J=8.4 \mathrm{~Hz}, 2 \mathrm{H}) ; 1782, \\
1745,1698,1280 \mathrm{~cm}^{-1}\end{array}$ & $\begin{array}{l}97 \\
93-94\end{array}$ & $\begin{array}{l}\delta=2.18(\mathrm{br}, 1 \mathrm{H}), 4.835(2 \mathrm{H}), 7.53(\mathrm{~d} \\
J=8.9 \mathrm{~Hz}, 2 \mathrm{H}), 8.21(\mathrm{~d}, J=8.8 \mathrm{~Hz}, 2 \mathrm{H}) \\
3517,1603,1509,1338,1057,736 \mathrm{~cm}^{-1}\end{array}$ \\
\hline $\begin{array}{l}\text { 4-Dimethylami- } \\
\text { nobenzamide (c)/B }\end{array}$ & $\begin{array}{l}95^{\mathrm{g}} \\
169-170 \\
2.18\end{array}$ & $\begin{array}{l}\delta=1.38(18 \mathrm{H}), 3.08(6 \mathrm{H}), 6.65(\mathrm{~d} \\
J=9.1 \mathrm{~Hz}, 2 \mathrm{H}), 7.76(\mathrm{~d}, J=9.1 \mathrm{~Hz}, 2 \mathrm{H}) \\
1785,1697,1595,1293,1099 \mathrm{~cm}^{-1}\end{array}$ & $\begin{array}{l}54 \\
\text { oil } \\
12^{\mathrm{g}} \\
180(\mathrm{dec})\end{array}$ & $\begin{array}{l}\delta=1.72(\mathrm{br}, 1 \mathrm{H}), 2.93(6 \mathrm{H}), 4.54(2 \mathrm{H}), 6.71 \\
(\mathrm{~d}, J=8.7 \mathrm{~Hz}, 2 \mathrm{H}), 7.22(\mathrm{~d}, J=8.7 \mathrm{~Hz}, 2 \mathrm{H}) \\
N \text {-Boc-4-dimethylaminobenzamide }(\text { side- } \\
\text { product }): \delta=1.53(9 \mathrm{H}), 3.04(6 \mathrm{H}), 6.65(\mathrm{~d}, \\
J=9.1 \mathrm{~Hz}, 2 \mathrm{H}), 7.72(\mathrm{~d}, J=9.1 \mathrm{~Hz}, 2 \mathrm{H}) ; E_{\mathrm{P}} / \\
\mathrm{V}=-2.26 \mathrm{~V}\end{array}$ \\
\hline 1-Naphthamide (d)/B & $\begin{array}{l}90^{\mathrm{g}} \\
67.5-68.5 \\
1.44\end{array}$ & $\begin{array}{l}\delta=1.26(18 \mathrm{H}), 7.48\left(\mathrm{dd}, J_{1}=8.2 \mathrm{~Hz},\right. \\
\left.J_{2}=7.2 \mathrm{~Hz}, 1 \mathrm{H}\right), 7.54 \text { and } 7.60(2 \times \mathrm{p} \mathrm{dt}, \\
\left.J_{1}=7 \mathrm{~Hz}, J_{2}=1.4 \mathrm{~Hz}, 1 \mathrm{H}+1 \mathrm{H}\right), 7.79 \\
\left(\mathrm{dd}, J_{1}=7.1 \mathrm{~Hz}, J_{2}=1.2 \mathrm{~Hz}, 1 \mathrm{H}\right), 7.89(\mathrm{p} \\
\mathrm{d}, J=8 \mathrm{~Hz}, 1 \mathrm{H}), 8.00(\mathrm{p} \mathrm{d}, J=8.2 \mathrm{~Hz}, 1 \\
\mathrm{H}), 8.37(\mathrm{p} \mathrm{d}, J=8 \mathrm{~Hz}, 1 \mathrm{H}) ; 1792,1736, \\
1717,1102 \mathrm{~cm}^{-1}\end{array}$ & $\begin{array}{l}86 / 51 \\
61-62\end{array}$ & $\begin{array}{l}\delta=1.95(1 \mathrm{H}), 5.09(2 \mathrm{H}), 7.41\left(\mathrm{dd}, J_{1}=8.1\right. \\
\left.\mathrm{Hz}, J_{2}=7.1 \mathrm{~Hz}, 1 \mathrm{H}\right), 7.47(\mathrm{p} \mathrm{d}, J=7 \mathrm{~Hz}, 1 \\
\mathrm{H}), 7.49 \text { and } 7.52\left(2 \times \mathrm{p} \mathrm{dt}, J_{1}=7 \mathrm{~Hz}, J_{2}=1.6\right. \\
\mathrm{Hz}, 1 \mathrm{H}+1 \mathrm{H}), 7.79(\mathrm{p} \mathrm{d}, J=8 \mathrm{~Hz}, 1 \mathrm{H}), 7.85 \\
\left(\mathrm{p} \mathrm{dd}, J_{1}=7.4 \mathrm{~Hz}, J_{2}=2 \mathrm{~Hz}, 1 \mathrm{H}\right), 8.06(\mathrm{p} \mathrm{d}, \\
J=8 \mathrm{~Hz}, 1 \mathrm{H}) ; 3368-3308(\mathrm{br}), 1003,800, \\
792,774 \mathrm{~cm}^{-1}\end{array}$ \\
\hline $\begin{array}{l}\text { Cyclohexanecarboxa- } \\
\text { mide }(\mathbf{e}) / \mathrm{B}\end{array}$ & $\begin{array}{l}74^{\mathrm{g}} \\
67-67.5 \\
-\end{array}$ & $\begin{array}{l}\delta=1.17-1.36 \text { and } 1.41-1.51(\mathrm{~m}, \mathrm{ca} .4 \mathrm{H}), \\
1.524(18 \mathrm{H}), 1.62-1.70(\mathrm{~m}, \mathrm{ca} .2 \mathrm{H}), 1.77 \\
\text { and } 1.81(2 \times \mathrm{p} \mathrm{t}, J=3 \mathrm{~Hz}, 1 \mathrm{H}+1 \mathrm{H}), \\
1.90 \text { and } 1.93(2 \times \mathrm{m}, 1 \mathrm{H}+1 \mathrm{H}), 3.16(\mathrm{tt}, \\
\left.J_{1}=11.3 \mathrm{~Hz}, J_{2}=3.4 \mathrm{~Hz}, 1 \mathrm{H}\right) ; 1777, \\
1745,1702 \mathrm{~cm}^{-1}, \text { estimated purity } \geq 95 \%\end{array}$ & $\begin{array}{l}\text { n.d. } \\
\text { oil }\end{array}$ & $\begin{array}{l}\delta=0.88-0.98(\mathrm{~m}, 2 \mathrm{H}), 1.11-1.32(\mathrm{~m}, 3 \mathrm{H}), \\
1.43-1.53(\mathrm{~m}, 1 \mathrm{H}), 1.63(1 \mathrm{H}), 1.65-1.78(\mathrm{~m}, \\
5 \mathrm{H}), 3.44(\mathrm{~d}, J=6.3 \mathrm{~Hz}, 2 \mathrm{H}) ; \text { ca. } 3290(\mathrm{br}), \\
2924,2854,1449,1036 \mathrm{~cm}^{-1}\end{array}$ \\
\hline $\begin{array}{l}\text { Boc-L-proline amide } \\
\text { (f)/A }\end{array}$ & $\begin{array}{l}85^{\mathrm{d}} \\
109-110 \\
-\end{array}$ & $\begin{array}{l}\delta=1.42 / 1.45(2 \times \mathrm{s}, 9 \mathrm{H}), 1.53 / 1.56 \\
(2 \times \mathrm{s}, 18 \mathrm{H}), 1.81-2.06 \text { and } 2.22-2.35 \\
(\mathrm{~m}, 4 \mathrm{H}), 3.37-3.49 \text { and } 3.51-3.64(\mathrm{~m}, 2 \\
\mathrm{H}), 5.11 / 5.19\left(2 \times \mathrm{dd}, J_{1}=8.9 \mathrm{~Hz},\right. \\
\left.J_{2}=3.5 / 2.7 \mathrm{~Hz}, 1 \mathrm{H}\right) ; 1774,1750,1708 \\
1697 \mathrm{~cm}^{-1}\end{array}$ & $\begin{array}{l}84 \\
59.5-60^{\mathrm{i}}\end{array}$ & $\begin{array}{l}\delta=1.47(9 \mathrm{H}), 1.56(\mathrm{~m}, 1 \mathrm{H}), 1.81(\mathrm{~m}, 2 \mathrm{H}), \\
1.99(\mathrm{~m}, 1 \mathrm{H}), 3.28-3.34 \text { and } 3.41-3.47(\mathrm{~m}, 1 \\
\mathrm{H}+1 \mathrm{H}), 3.61(\mathrm{br}, 2 \mathrm{H}), 3.95(\mathrm{br}, 1 \mathrm{H}) ; 3438 \\
1664,1412,1049 \mathrm{~cm}^{-1}\end{array}$ \\
\hline
\end{tabular}

a Yields are not optimized.

${ }^{\text {b }}{ }^{1} \mathrm{H}$ NMR spectra recorded at $400 \mathrm{MHz}$ in $\mathrm{CDCl}_{3}$. Proton signals are singlets, when not otherwise stated. Major/minor conformer. FT-IR spectra recorded in $\mathrm{KBr}$ pellets (for 3a and 3e as films). Only carbonyl and hydroxyl and occasionally strongest bands given. Abbreviation: $\mathrm{p}=$ perturbed.

${ }^{\mathrm{c}}$ Solvents used in the preparation of 2: acetonitrile (A), dichloromethane (B).

${ }^{\mathrm{d}}$ Previously described compound (ref. ${ }^{7}$ ).

e Twice chromatographed on silica gel with $\mathrm{Et}_{2} \mathrm{O} /$ light petroleum 1:4 (traces of $\mathrm{HNBoc}_{2}$ still visible).

${ }^{f}$ See ref. ${ }^{1 b}$

g Novel compound with satisfactory $\mathrm{C}, \mathrm{H}, \mathrm{N}$ analysis.

${ }^{\mathrm{h}}$ Chromatographed on silica with $\mathrm{Et}_{2} \mathrm{O} / \mathrm{CH}_{2} \mathrm{Cl}_{2}$ 1:15 but strongly contaminated with Boc-compound(s).

${ }^{\mathrm{i}}[\alpha]_{\mathrm{D}}{ }^{25}-53.8$ and $[\alpha]_{578}{ }^{25}-56.2(c 1.0, \mathrm{MeOH})$, the former value in agreement with literature. 


\section{Acknowledgment}

This work was supported by Magn. Bergvall's stiftelse (U.R.), O.E. och E. Johansson's stiftelse and Helge Ax:son Johnson's stiftelse (L.G.) and FCT (Portugal) (L.S.M. and H.L.S.M.). U.R. also thanks the RSC for a journals grant for international authors.

\section{References}

(1) (a) Ragnarsson, U.; Grehn, L.; Maia, H. L. S.; Monteiro, L. S. Org. Lett. 2001, 3, 2021. (b) Ragnarsson, U.; Grehn, L.; Maia, H. L. S.; Monteiro, L. S. J. Chem. Soc., Perkin Trans. $12002,97$.

(2) (a) For an overview: Barrett, A. G. M. In Comprehensive Organic Synthesis. Selectivity, Strategy and Efficiency in Modern Organic Synthesis, Vol. 8; Trost, B. M.; Fleming, I., Eds.; Pergamon: Oxford, 1991, 248-251. (b) Kamochi, Y.; Kudo, T. Chem. Pharm. Bull. 1994, 42, 402.

(3) (a) Fisher, G. B.; Fuller, J. C.; Harrison, J.; Alvarez, S. G.; Burkhardt, E. R.; Goralski, C. T.; Singaram, B. J. Org. Chem. 1994, 59, 6378. (b) Myers, A. G.; Yang, B. H.; Kopecky, D. J. Tetrahedron Lett. 1996, 37, 3623.

(4) Reviews: (a) Hoefle, G.; Steglich, W.; Vorbrueggen, H. Angew. Chem., Int. Ed. Engl. 1978, 17, 569; Angew. Chem. 1978, 90, 602. (b) Hassner, A. In Encyclopedia of Reagents for Organic Synthesis; Paquette, L. A., Ed.; Wiley:

Chichester, 1995, 2022. (c) Ragnarsson, U.; Grehn, L. Acc. Chem. Res. 1998, 31, 494.

(5) Flynn, D. L.; Zelle, R. E.; Grieco, P. A. J. Org. Chem. 1983, 48, 2424.

(6) Grehn, L.; Ragnarsson, U. Angew. Chem., Int. Ed. Engl. 1985, 24, 510; Angew. Chem. 1985, 97, 519.

(7) Davidsen, S. K.; May, P. D.; Summers, J. B. J. Org. Chem. 1991, 56, 5482.
(8) Maia, H. L. S.; Monteiro, L. S.; Degerbeck, F.; Grehn, L.; Ragnarsson, U. J. Chem. Soc., Perkin Trans. 2 1993, 495.

(9) Typical Reduction Procedure: A solution of 2 c (732 mg, 2 $\mathrm{mmol})$ in abs EtOH $(12 \mathrm{~mL})$ was treated with an excess of $\mathrm{NaBH}_{4}(151 \mathrm{mg}, 4 \mathrm{mmol})$ in one batch. After $20 \mathrm{~h}$, when all starting material had been consumed (TLC monitoring), acetone $(1 \mathrm{~mL})$ was added, whereupon after $1 \mathrm{~h}$ the solvent was evaporated. The obtained foam was dissolved in $\mathrm{Et}_{2} \mathrm{O} /$ $\mathrm{H}_{2} \mathrm{O}$, the aqueous phase backwashed once with $\mathrm{Et}_{2} \mathrm{O}$ and the combined extracts dried $\left(\mathrm{Na}_{2} \mathrm{SO}_{4}\right)$. After evaporation the mixture was purified by chromatography on silica gel (eluent: $\mathrm{Et}_{2} \mathrm{O} / \mathrm{CH}_{2} \mathrm{Cl}_{2}$ 1:15). $\mathrm{HNBoc}_{2}$ eluted first, followed by pure $3 \mathbf{c}(298 \mathrm{mg}, 97 \%)$; $\mathrm{mp} 91-92{ }^{\circ} \mathrm{C}$; it was recrystallized from $\mathrm{Et}_{2} \mathrm{O}$ /light petroleum.

(10) Degerbeck, F.; Grehn, L.; Ragnarsson, U. Acta Chem. Scand. 1993, 47, 896.

(11) Greene, T. W.; Wuts, P. G. M. Protective Groups in Organic Synthesis, 3rd ed.; Wiley-Interscience: New York, 1999, 518.

(12) (a) Kim, B. M.; Evans, B. E.; Gilbert, K. F.; Hanifin, C. M.; Vacca, J. P.; Michelson, S. R.; Darke, P. L.; Zugay, J. A.; Emini, E. A.; Schleif, W.; Lin, J. H.; Chen, I.-W.; Vastag, K.; Anderson, P. S.; Huff, J. R. Bioorg. Med. Chem. Lett. 1995, 5, 2707. (b) Ennis, M. D.; Ghasal, N. B.; Hoffman, R. L.; Smith, M. W.; Schlachter, S. K.; Lawson, C. F.; Im, W. B.; Pregenzer, J. F.; Svensson, K. A.; Lewis, R. A.; Hall, E. D.; Sutter, D. M.; Harris, L. T.; McCall, R. B. J. Med. Chem. 1998, 41, 2180. (c) Kojima, N.; Minakawa, N.; Matsuda, A. Tetrahedron 2000, 56, 7909. (d) Vedejs, E.; Kongkittingam, C. J. Org. Chem. 2001, 66, 7355. (e) Perron-Sierra, F.; Saint Dizier, D.; Bertrand, M.; Genton, A.; Tucker, G. C.; Casara, P. Bioorg. Med. Chem. Lett. 2002, 12, 3291.

(13) Smith, M. B.; March, J. March's Advanced Organic Chemistry. Reactions, Mechanisms, and Structure, 5th ed.; Wiley: New York, 2001, 1546. 